\title{
The effect of stimulus intensity on motor latency in the carpal tunnel syndrome
}

\author{
GEORGE PRESWICK ${ }^{1}$ \\ From the Department of Applied Electrophysiology, National Hospital, Queen Square, London
}

In the measurement of motor latency it is customary to stimulate the nerve trunk with a shock that is strong enough to produce a maximal response. Thus the latency, measured to the first deflection of the muscle action potential, depends on the fastest conducting fibres in the nerve trunk. In a partial nerve lesion this means that a few unaffected fibres will be sufficient to give a normal latency despite the presence of other damaged fibres with reduced conduction velocities. As a diagnostic test, therefore, the measurement of latency to supramaximal stimulation lacks sensitivity and may give a normal result in spite of clinical evidence to the contrary. For example, in an unselected series of 95 consecutive cases with a diagnosis of suspected carpal tunnel syndrome, Thomas (1960) was able to demonstrate an abnormal motor latency to supramaximal stimulation in only two-thirds of the cases.

It is not an uncommon observation in patients with a carpal tunnel syndrome that an isolated motor unit potential with a long latency may appear in response to a threshold stimulus and subsequently become obscured by additional complexes as the stimulus intensity is increased. It was the purpose of this study to investigate the diagnostic significance of these 'late' motor units in the carpal tunnel syndrome.

\section{MATERIAL AND METHODS}

All examinations were performed in a warm room. The subjects lay on a couch with the arm positioned comfortably on a pillow alongside the couch.

A co-axial needle electrode was inserted into the belly of the abductor pollicis brevis muscle and its position later adjusted to provide the largest muscle action potential at threshold stimulation.

For stimulation, small pad electrodes approximately $1 \mathrm{~cm}$. in diameter were used. The stimulating cathode was placed over the course of the median nerve at a point about $1 \mathrm{~cm}$. above the distal wrist crease and its

'Present address: Department of Neurology, Prince Henry Hospital, Little Bay, Sydney, Australia position changed when necessary to give the best stimulation of the motor fibres as judged by the least stimulating voltage required to produce a threshold response. The anode was an electrode of similar size placed over the course of the median nerve $5 \mathrm{~cm}$. above the distal wrist crease. The conduction distance was measured on the skin from the centre of the stimulating cathode to the point of entry of the recording needle. This varied in different subjects between 5 and $6.5 \mathrm{~cm}$.

The stimulus was a brief condenser discharge (time constant $100 \mu \mathrm{sec}$.); it was delivered once per second through a $1: 1$ isolating transformer (output impedance $1,000 \mathrm{ohms})$. The voltage was continuously variables up to 375 volts.

The muscle action potentials were amplified by $\mathbb{\mathbb { Q }}_{\mathbb{2}}$ R-C coupled amplifier and displayed on a double-beamo cathode ray oscilloscope, the second beam of which provided a time scale with 1 and $5 \mathrm{msec}$. intervals Muscle action potentials were always examined using high amplification to ensure that small early deflectionso were not overlooked. The stimulus was triggered at a fixed point on the time base of the oscilloscope and the whole display was photographed on $70 \mathrm{~mm}$. photographic paper. The motor latency was then measured from the beginning of the stimulus artefact to the first deflection of the muscle action potential.

In a few patients, sensory nerve conduction was also examined. The stimulus was applied to the index finger, and action potentials were recorded from the median nerve at the wrist, using a technique similar to that described by Gilliatt and Sears (1958).

In order to ensure that differences in nerve threshold and motor latency between control subjects and carpal tunnel patients were not due to differences in limb temperature, skin temperature over the thenar eminence was recorded by a surface thermistor in all the control subjects and in eight of the patients. In another 13 patients muscle temperature was measured by a needle thermistor buried in the hypothenar eminence. The mean skin temperature record in the control group was $31 \cdot 1^{\circ} \mathrm{C}$. (range $26.7^{\circ} \mathrm{C}$. to $34.6^{\circ} \mathrm{C}$.) and in the patients it was $30.9^{\circ} \mathrm{C}$. (range $26.4^{\circ} \mathrm{C}$. to $34 \cdot 6^{\circ} \mathrm{C}$.). The mean muscle temperature in the patients was $32.8^{\circ} \mathrm{C}$. (range $27 \cdot 2^{\circ} \mathrm{C}$. to $35.4^{\circ} \mathrm{C}$.) which may be compared with muscle temperatures ranging from $34.0^{\circ} \mathrm{C}$. to $36 \cdot 5^{\circ} \mathrm{C}$, , recorded from the proximal third of the forearm by Henriksen (1956). 
RESULTS

CONTROL SUBJECTS Motor latency with threshold and with supramaximal stimulation was estimated in 25 control subjects who were approximately matched for sex and age with the patients. Four of these subjects were suffering from neurological disorders which did not affect peripheral nerves; the remainder were healthy adult subjects drawn from the hospital staff. All but two of the subjects were women and the age range was 33 to 70 years. The results are shown in Figure 1. With threshold stimulation, motor latency varied in different individuals from 2.6 to $5.2 \mathrm{msec}$. with a mean of 3.88 msec. (S.D. \pm 0.53 ). With supramaximal stimulation, the range was $2 \cdot 1$ to $4.7 \mathrm{msec}$. with a mean of $3.33 \mathrm{msec}$. (S.D. \pm 0.46 ). The difference of these means is highly significant $(P<0.001)$.

PATIENTS WITH SUSPECTED CARPAL TUNNEL SYNDROME All the patients used in this study had been referred for electromyography by Professor R. W. Gilliatt with a clinical diagnosis of carpal tunnel syndrome. Twenty-five patients were referred, and in four of these the condition was thought to be bilateral; thus 29 hands were examined. All the patients except one were women, with an age range of 34 to 71 years. As the object of the investigation was to study the early changes in motor conduction, most of the cases examined were only mildly affected clinically. Only half the patients exhibited abnormal physical signs, and in the majority of these, mild sensory impairment in the territory of the median nerve was the only abnormality. In most of the patients the duration of symptoms was two years or less but in four patients there had been intermittent symptoms for over 10 years.

Motor latency with threshold and with supramaximal stimulation was estimated in all patients and the results for the whole group are shown in Figure 1. It can be seen that motor latency with supramaximal stimulation was normal in 11 out of 29 cases whereas motor latency with threshold stimulation was normal in only five cases. Some degree of weakness and wasting of the abductor pollicis brevis muscle was present in seven cases; in these, motor latency with supramaximal stimulation was abnormal in six cases while the latency with threshold stimulation was abnormal in all of them. There were 10 cases without motor signs but with sensory impairment in the median-supplied digits; in this group, motor latency was abnormal with supramaximal stimulation in eight cases whereas threshold stimulation gave abnormal latencies in all cases. In 12 cases the diagnosis rested on the patient's history, no evidence of median nerve damage being found on physical examination. In this group, motor latency was normal with supramaximal stimulation in eight cases and with threshold stimulation in five. There were thus five patients in whom motor latencies were normal with both threshold and supramaximal stimulation. In four of these patients sensory nerve conduction was also examined and appeared to be normal by the criteria of Gilliatt and Sears (1958). On reviewing the history in these five cases, there were two in

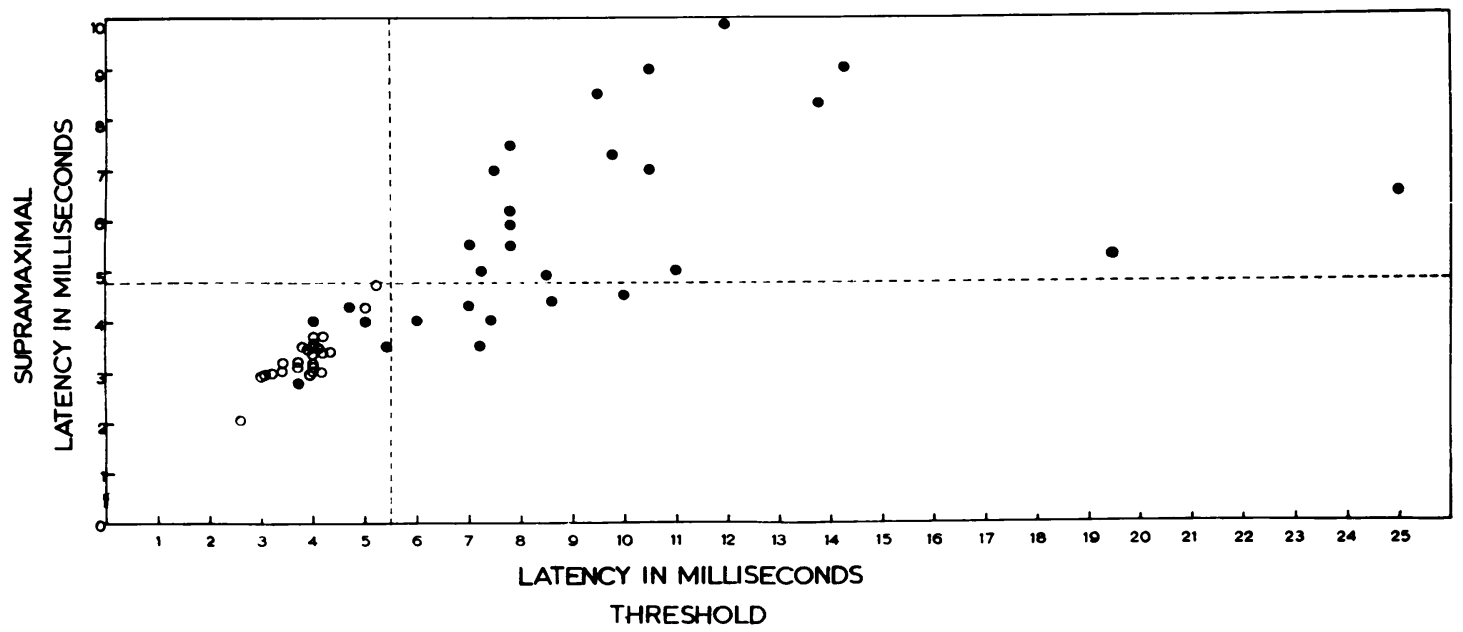

FIG. 1. Latency of the motor response from the abductor pollicis brevis with threshold and supramaximal stimulation of the median nerve at the wrist in patients with the carpal tunnel syndrome (filled circles) and control subjects (open circles). The broken lines indicate the upper limits of normal motor latency (mean +3 standard deviations). 
which the diagnosis was subsequently considered to be uncertain. In the remaining three cases the history was typical of the carpal tunnel syndrome although no confirmatory physical or electrical abnormalities were detected.

A typical result from one of the more severely affected patients is illustrated in Figure 2.

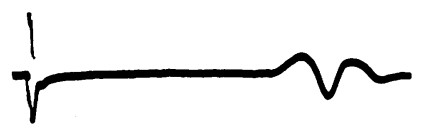

$77 \mathrm{~V}$

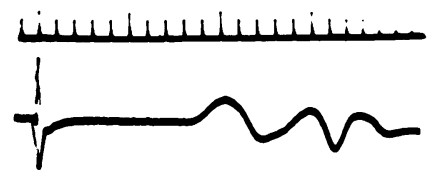

$83 \mathrm{~V}$.

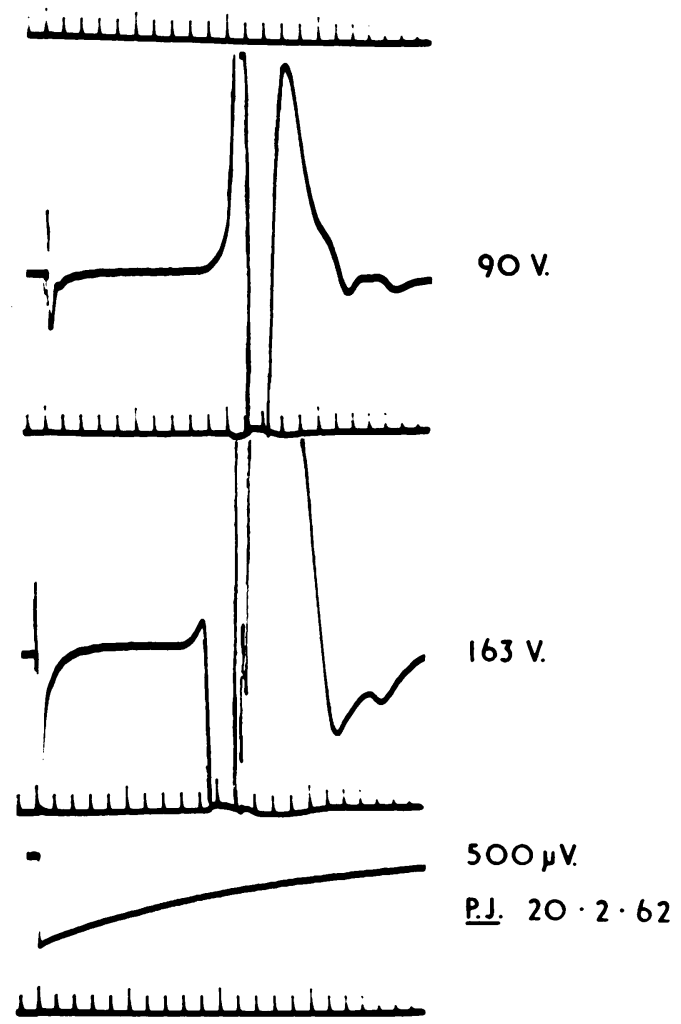

FIG. 2. Action potentials recorded from the right abductor pollicis brevis in a patient $(P . J$.$) with the carpal tunnel$ syndrome. Motor latency is progressively shortened as the stimulus intensity is increased from threshold (top) to supramaximal (bottom). Time scale: 1 and 5 msec. intervals.
The patient (P.J.), a housewife, aged 52, had a two-year history of intermittent pain in the hands, occurring mostly at night and associated with a feeling of numbness and swelling of the fingers, except the little finger, on each side. On examination there was moderate weakness and wasting of the abductor pollicis brevis muscle on the right side, together with slight impairment of sensation to pin-prick in the distribution of the median nerve on this side. A threshold stimulus ( 77 volts) applied over the median nerve at the wrist produced a motor action potential with a latency of $13.8 \mathrm{msec}$.; on increasing the stimulus intensity additional action potentials appeared so that, with a supramaximal stimulus (163 volts), the latency was reduced to $8.3 \mathrm{msec}$. The examination was repeated on this patient seven weeks later, during which time no treatment had been given, and almost identical motor latencies were observed.

STIMULATING VOLTAGES Absolute figures for the voltages employed to stimulate the median nerve showed considerable variation in different individuals in both the control and patient groups, presumably due to factors such as skin resistance and thickness of the subcutaneous tissues. In spite of this, the mean threshold voltages were remarkably similar for the two groups, being 95 volts for the controls an 97 volts for the patients. The mean voltage require $\$$ to produce a maximal response was only slightlo higher in the patients (168 volts) than in the controls (152 volts) and it appears that, with a stimulus applied above the wrist crease, as used in this study a clearly raised nerve threshold should only bs expected in very advanced cases.

\section{DISCUSSION}

Conduction in the distal part of the median nerve was first studied by Simpson (1956) who considered that with supramaximal stimulation of the nerve at the wrist the normal motor latency should be less than $5 \mathrm{msec}$. For recording the muscle action potential, Simpson used mainly the opponens pollicis muscle whereas subsequent workers have preferred the abductor pollicis brevis on the grounds that the latter muscle is more likely to receive its entire nerve supply from the median nerve (Rowntree, 1949). In the 50 control subjects examined by Thomas (1960), the mean motor latency for the abductor pollicis brevis with supramaximal stimulation of the median nerve at the wrist was $3.8 \mathrm{msec}$. \pm 0.5 . This is significantly longer $(P<0.001)$ than the mean value for the 25 control subjects $(3.3 \mathrm{msec}$. $\pm 0.5)$ in the present series. The difference is most likely to be due to the shorter conducting distance employed in the present series $(5-6.5 \mathrm{~cm}$. as against $6-8 \mathrm{~cm}$. in Thomas's series). 
Limb temperature is known to affect motor latency (Carpendale, 1956) and while there is no reason to suppose that Thomas's control subjects were colder than those in the present series the lack of temperature data in the former makes it impossible to exclude this possibility.

In any study involving the measurement of latency with supramaximal stimulation, the possibility of distal spread of the stimulating current from under the cathode must be considered, particularly when using strong shocks, as this may give the false impression of more rapid conduction in the nerve. Henriksen (1956) discussed this question in detail and concluded that it was not an important factor when relatively large electrodes were used for percutaneous stimulation. Some distal spread of current at high stimulating intensities cannot be excluded in the present experiments and indeed may have contributed to the shortening of motor latency with supramaximal, as compared with threshold, stimulation in the control subjects. It cannot, however, have been a significant factor in the patients in whom latency changes of several milliseconds occurred as a result of only small changes in stimulus intensity.

The duration of the summated action potential from the thenar muscles with stimulation of the median nerve at the wrist rarely exceeds $20 \mathrm{msec}$. in normal subjects (Simpson, 1956; Christie and Coomes, 1960). In the carpal tunnel syndrome the duration of the summated action potential is often prolonged (Simpson, 1956) and this is regarded by Bauwens (1958) as a valuable sign of nerve damage. There is, however, difficulty in these cases in measuring the extent of the temporal dispersion because of the possible contribution of repetitive firing, which occurred in nearly half of Simpson's original cases. Stimulating at threshold circumvents this difficulty and makes it possible to study delayed motor units in isolation.

Recording of motor latency with threshold stimulation has not been extended to conditions other than the carpal tunnel syndrome, and while it has been shown to be a sensitive method of demonstrating early evidence of nerve damage, it should not be supposed that it is specific to this disorder as it is likely that delayed motor units will be found in any condition associated with differential slowing of nerve conduction.

It is interesting to note that, of the 10 patients who had only sensory symptoms and signs, nearly all showed abnormalities with threshold stimulation; this raises the question of whether a higher proportion of electrical abnormalities would have been found in mildly affected patients by examining sensory conduction. This was not studied systematically in the present series but, as it is known that the degree of involvement of motor and sensory nerve fibres may vary independently in the carpal tunnel syndrome, the measurement of motor latency at threshold should not be regarded as making the study of sensory conduction unnecessary.

\section{SUMMARY}

Motor latency has been studied in a selected series of 25 patients with a clinical diagnosis of carpal tunnel syndrome, using threshold and supramaximal stimulation of the median nerve at the wrist.

Most of the cases were only mildly affected clinically and less than half showed abnormal latencies with supramaximal stimulation, whereas isolated motor units with prolonged latencies were present with threshold stimulation in over $80 \%$ of cases.

It is concluded that the study of motor latency with threshold stimulation is of value in detecting early evidence of nerve damage.

I wish to record my gratitude for the help and encouragement given by Professor Gilliatt who also referred all the patients used in this study. I also wish to thank Dr. W. A. Cobb for helpful criticism.

\section{REFERENCES}

Bauwens, P. (1958). Proc. roy. Soc. Med., 51, 725.

Carpendale, M. T. F. (1956). M.S. (Phys. Med.) Thesis, University of Minnesota.

Christie, B. G. B., and Coomes, E. N. (1960). Ann. phys. Med., 5, 303.

Gilliatt, R. W., and Sears, T. A. (1958). J. Neurol. Neurosurg. Psychiat., 21, 109.

Henriksen, J. D. (1956). M.S. (Phys. Med.) Thesis, University of Minnesota.

Rowntree, T. (1949). J. Bone Jt Surg., 31B, 505.

Simpson, J. A. (1956). J. Neurol. Neurosurg. Psychiat., 19, 275.

Thomas, P. K. (1960). Neurology (Minneap.), 10, 1045. 